\title{
Impact of lung-RADS classification system on the accurate diagnosis of pulmonary nodular lesions in oncology patients
}

\author{
Hoda Abdel Kareem Ahmed ${ }^{1 *}$ and Mohamed FarghalyAmin ${ }^{2}$
}

\begin{abstract}
Background: Lung assessment is highly recommended in the management of oncology patients as it is the commonest affected site in metastatic dissemination. The low-dose CT with nodule reporting system based on Lung Reporting and Data System (lung-RADS) is a promising non-invasive tool for the characterization of incidentally detected pulmonary nodules. The authors aimed to assess the accuracy of the "lung-RADS" classification system as a non-invasive tool for the characterization of any newly developed pulmonary nodules among oncology patients. Ethics committee approval and informed written consent were obtained from the studied patients. A noncontrast LDCT study was performed on all patients with a nodule reporting system based on the lung-RADS classification system applied for evaluation of each detected pulmonary nodule. Diagnoses were established using the help of either histopathology or follow-up clinical results as a gold standard.

Results: In this prospective study, we enrolled 187 known malignancy patients with 200 suspicious newly developed pulmonary nodules. Their mean patient age was $48.4 \pm 9.7$ years. The studied 200 pulmonary nodular lesions were categorized using a nodule reporting system based on the lung-RADS into 6 sub-groups with 122 lesions found to be malignant and 78 lesions were of benign etiology, which showed a high sensitivity of 92.08\%, specificity of $78.79 \%$, and accuracy of $85.50 \%$ with $81.58 \%$ positive predictive value and $90.70 \%$ negative predictive value in the diagnosis of pulmonary nodules in cancer patients.
\end{abstract}

Conclusion: Low-density CT with a nodule reporting system based on the lung-RADS classification system was found to be an accurate non-invasive tool to characterize and to risk stratify pulmonary nodules in oncology patients.

Keywords: Lung-RADS, Pulmonary nodules, Oncology

\section{Background}

In daily clinical practice, radiologists and pulmonologists are faced with incidental findings of pulmonary nodules that require an optimal decision in management and, meanwhile, to avoid morbidity and increased costs which are caused by invasive and unwarranted procedures for benign disease [1]. The differential diagnosis for pulmonary nodules is extremely broad, including

\footnotetext{
*Correspondence: Hodaradiology2012@gmail.com

${ }^{1}$ Radio-Diagnosis Department, Faculty of Medicine, Al-Azhar University, Assuit, Egypt

Full list of author information is available at the end of the article
}

both benign and malignant causes [2]. There is no consensus or guidelines regarding the investigation of pulmonary nodules in patients with extra-pulmonary malignancies. Retrospective data in populations of patients with cancer shows a high frequency of benign lesions (up to 58\%) and primary lung cancers (up to 50\%) found after biopsies or surgery [3]. Computed tomography $(\mathrm{CT})$ is the most widely used modality for detecting lung metastases [4]. While the interpretation of CT scans for lung screening in pulmonary nodules remains challenging, various protocols and scoring systems have been developed [5]. The Reporting and Data System

\section{Springer Open}

(c) The Author(s). 2021 Open Access This article is licensed under a Creative Commons Attribution 4.0 International License, which permits use, sharing, adaptation, distribution and reproduction in any medium or format, as long as you give appropriate credit to the original author(s) and the source, provide a link to the Creative Commons licence, and indicate if changes were made. The images or other third party material in this article are included in the article's Creative Commons licence, unless indicated otherwise in a credit line to the material. If material is not included in the article's Creative Commons licence and your intended use is not permitted by statutory regulation or exceeds the permitted use, you will need to obtain permission directly from the copyright holder. To view a copy of this licence, visit http://creativecommons.org/licenses/by/4.0/. 
Table 1 Summary of Lung-RADS category [9]

\begin{tabular}{|c|c|c|c|}
\hline $\begin{array}{l}\text { Category } \\
\text { descriptor }\end{array}$ & $\begin{array}{l}\text { Category } \\
\text { malignancy }\end{array}$ & $\begin{array}{l}\text { Probability } \\
\text { management }\end{array}$ & Recommendation \\
\hline Negative & 1 & $<1 \%$ & Continue annual screening with LDCT in 12 months \\
\hline $\begin{array}{l}\text { Benign appearance } \\
\text { or behavior }\end{array}$ & 2 & $<1 \%$ & Continue annual screening with LDCT in 12 months \\
\hline Probably benign & 3 & $1-2 \%$ & 6-month LDCT \\
\hline \multirow[t]{2}{*}{ Suspicious } & $4 \mathrm{~A}$ & $5-15 \%$ & 3-month LDCT; PET/CT may be used when there is a $\geq 8 \mathrm{~mm}$ solid component \\
\hline & $\begin{array}{l}4 B \\
4 X\end{array}$ & $>15 \%$ & $\begin{array}{l}\text { Chest } C T \text { with/without contrast, } \mathrm{PET} / \mathrm{CT} \text {, and/or tissue sample that depends on the } \\
\text { probability of malignancy and comorbidities. PET-CT may be used when there is a } \geq 8 \text {-mm } \\
\text { solid component }\end{array}$ \\
\hline
\end{tabular}

(lung-RADS) was introduced to standardize the reporting and management of patients undergoing screening to facilitate data collection and monitoring of patient outcomes [6]. In this system, a specific lung-RADS category is assigned to each low-dose computed tomography (LDCT) examination. Each category measures the likelihood of malignancy for the dominant finding and offers specific recommendations regarding management [7]. The primary goal of lung-RADS is to minimize variation in the management of detected lung nodules that can be implemented effectively in radiology practices outside the purview of a clinical trial [8].

The purpose of the work was to assess the accuracy of the "lung-RADS" classification system as a non-invasive tool for characterization of any newly developed pulmonary nodules among oncology patients using the help of either histopathology or follow-up clinical results as a gold standard.

\section{Methods}

\section{Patients' demographic data}

This prospective cross-sectional analytic study included 187 patients (109 females and 78 males) with a known history of malignancy who are under management and followed up with 200 newly developed pulmonary nodules that are suspicious of a metastatic etiology. The

Table 2 Demographics

\begin{tabular}{lll}
\hline Variable & Overall & Percent (\%) \\
\hline Number & 187 pts with 200 nodules & $100 \%$ \\
Age & $21-78( \pm 48.4)$ years & - \\
Sex & 109 females & $58.28 \%$ \\
& 78 males & $41.71 \%$ \\
Known primary neoplasm & \\
-Breast Ca & 83 & $41.5 \%$ \\
-Lung Ca & 68 & $34 \%$ \\
-Prostate Ca & 19 & $9.5 \%$ \\
-GIT Ca & 12 & $6 \%$ \\
-Thyroid Ca & 5 & $2.5 \%$ \\
\hline
\end{tabular}

patient's age ranged from 21 to 78 years old with the mean age $48.4 \pm 9.7$ years.

Ethics committee approvals in addition to informed written consent were obtained from all patients.

\section{Inclusion criteria}

Patients with a known history of treated malignancy (either surgical, radio, or chemo-therapy) with newly developed pulmonary nodule/s on a follow-up CT chest study were included in the study.

Patients who had general contraindications for CT examination or respiratory distress that made them unable to tolerate breath-hold were excluded.

\section{CT protocol and technique}

The non-contrast LDCT study was performed using a 16-Multislice CT scanner (GE) Aquilion machine. All patients were trained to achieve a full end-inspiratory breath-hold before the start of the LDCT exam. They were scanned at the end of the full inspiration CT protocol: $120-140 \mathrm{kVp}, 40-100 \mathrm{~mA}$, and reconstructed section width 1.0 to $3.2 \mathrm{~mm}$, with reconstruction scan intervals of $1-2.5 \mathrm{~mm}$ and a pitch of $1-2$.

\section{Post-processing}

All CT images were magnified and reviewed by two radiologists who had prior experience with chest imaging and were informed of the patient's history, using GE PACS in axial images and reconstructed with soft tissue and lung windows. Axial maximum-intensity projections $(16-2.5 \mathrm{~mm})$. Coronal and sagittal multiplanar reformatted images were reconstructed and used for interpretation. The window setting for lung parenchyma used was between -560 and -1135 HU by the visual method to detect any pulmonary nodular lesions as well as the associated parenchymal abnormalities. According to the lung-RADS classification system (Table 1), nodules can be categorized into $1-4 \mathrm{~A}, \mathrm{~B}$, and $\mathrm{X}$ subgroups [9]. CT image interpretation was performed by two radiologists in consensus with adequate experience in CT lung screening using lung-RADS guidelines and nodule 
Table 3 Final clinico-pathological diagnosis results of the all studied nodular lesions (no. = 200)

\begin{tabular}{llll}
\hline Final lesion clinical/pathology diagnosis & & No. & Percent \% \\
\hline Group I: Benign lesions & & $\mathbf{7 8}$ & $39 \%$ \\
a) Ground glass nodules & $\mathbf{2 6 / 0}$ & 26 & $13 \%$ \\
b) Pre-fissural nodules & $\mathbf{1 7 / 0}$ & 17 & $8.5 \%$ \\
c) Inflammatory nodules & $\mathbf{1 3 / 0}$ & 13 & $6.5 \%$ \\
d) Follow-up static small nodules & $\mathbf{1 0 / 0}$ & 10 & $5 \%$ \\
e) Fat/calcifications containing nodules & $\mathbf{1 2 / 0}$ & 12 & $6 \%$ \\
Group Il; Malignant lesions & & $\mathbf{1 2 2}$ & $61 \%$ \\
a) Metastatic nodules & $\mathbf{1 9 / 7 8}$ & 87 & $43.5 \%$ \\
b) Sequamus cell carcinoma & $\mathbf{0 / 1 9}$ & 19 & $9.5 \%$ \\
c) Adeno-carcinoma & $\mathbf{0 / 7}$ & 7 & $3.5 \%$ \\
d) Alveolar lung cancer & $\mathbf{0 / 6}$ & 6 & $3 \%$ \\
e) Small cell carcinoma & $\mathbf{0 / 3}$ & 3 & $1.5 \%$ \\
Total & & 200 & $100 \%$ \\
\hline
\end{tabular}

follow-up algorithms. Nodules are considered positive for malignancy and are categorized as $4 \mathrm{~A}, 4 \mathrm{~B}$, and $4 \mathrm{X}$.

\section{Assessment of nodule size and density}

On CT images, each pulmonary nodule was studied for:

\section{The lesion size is given in millimeters}

Leurion texture, which is expressed as soft tissue attenuation "solid," sub-solid, which could be divided into either pure ground-glass attenuation with pulmonary vessels and bronchi that may be seen within, or a semi-solid/part-solid that contains both ground glass and soft-tissue attenuation components. The categorization of a pulmonary nodule is important in that it predicts the likelihood of malignancy.

Histo-pathological and final clinical diagnosis as a gold standard

CT findings with the lung-RADS classification system were correlated by either histopathological results for very suspicious large malignant lesions or follow-up of final clinical diagnosis for benign featuring small nodular lesions in all patients. The nodules were classified as benign or malignant based on the final diagnosis.

\begin{abstract}
Statistical analysis
The statistical calculations were done using the Statistical Package for Social Science, version 19, in which data is described in terms of range, mean, standard deviation, frequencies \& percentages. Sensitivity, specificity, accuracy, positive predictive value, and negative predictive value for $\mathrm{CT}$ results with a lung-RADS classification system for pulmonary nodules were calculated.
\end{abstract}

\section{Results}

This prospective study included 187 patients who had a positive history of treated cancer ( 83 breast cancer, 68 lung cancer, 19 prostate cancer, 12 GIT cancer, and 5 thyroid cancer) and were under management and follow-up with 200 newly developed pulmonary nodules that were suspicious of metastatic etiology. The patients' ages ranged from 21 to 78 years $(M=43.4 \pm 9.7$ years $)$ (Table 2).

\section{Gold standard}

The histopathological results for the biopsed103 nodules were as follows: 72 metastatic nodules, 9 Seq. cell $\mathrm{Ca}, 7$ adeno- $\mathrm{Ca}, 6$ alveller $\mathrm{Ca}, 3$ small cell $\mathrm{Ca}$, and 6 inflammatory lesions, while the final clinical diagnoses based on the clinical and follow-up (for 18-24 months) results of the remaining 97 inaccessible small/benign featuring lesions were as follows: 25 metastatic lesions that showed either increase in size or number in follow-up CT images. However, 72 lesions were categorized as benign nodules which were stationary on CT follow-up studies up to 2 years duration. Table 3 shows the classification of all pulmonary nodular lesions studied $($ no $=200)$ into two groups: group I: benign nodular lesions (78 lesions; 39\%) and group II: malignant nodular lesions (122 lesions; 61\%).

The diagnosis of lung RADS necessitates the examination of 200 nodules

Eighty-six lesions were diagnosed as benign (score 1-3), although 8 of them were found to be malignant

Table 4 Categorization of benign lung nodules (lung-RRADS score 1-3): "group I" (no. = 86 nodules)

\begin{tabular}{|c|c|c|c|c|c|}
\hline RADS score & Size & Lesion density & Lesion no. & $\%$ for all lesions & Radio-diagnosis \\
\hline \multirow[t]{2}{*}{1} & Any & Fully-pop corn calcification & 13 & $6.5 \%$ & Benign \\
\hline & Any & Fatty containing & 5 & $2.5 \%$ & \\
\hline \multirow[t]{2}{*}{2} & $6-<30 \mathrm{~mm}$ & Solid pre-fissural/+ground glass appearance & 29 & $14.5 \%$ & Benign \\
\hline & $<6 \mathrm{~mm}$ & Sub-solid/semi-solid & 13 & $6.5 \%$ & \\
\hline \multirow[t]{3}{*}{3} & $\geq 6$ to $<8 \mathrm{~mm}$ & Solid & 17 & $8.5 \%$ & Benign \\
\hline & $\geq 6 \mathrm{~mm}$ with solid component $<6 \mathrm{~mm}$ & Part solid & 6 & $3 \%$ & \\
\hline & $\geq 30 \mathrm{~mm}$ & Non-solid & 3 & $1.5 \%$ & \\
\hline
\end{tabular}




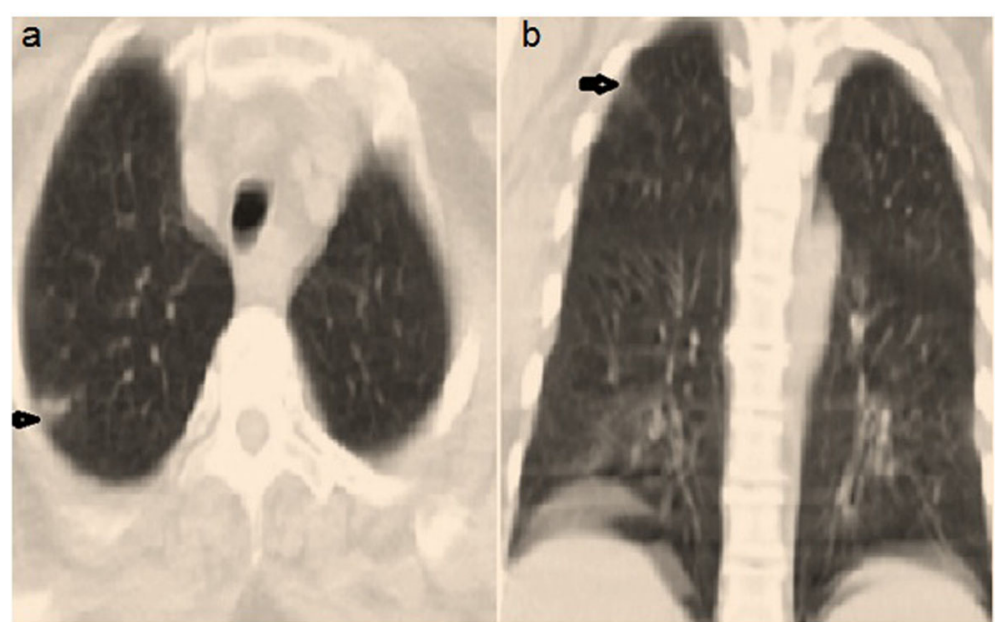

Fig. 1 "A 47-year-old male patient with a history of rectum cancer." MCCT exam revealed a small well-defined solid nodule about $6 \times 3 \mathrm{~mm}$ at the posterior aspect of the Rt. Upper lung lobe (a) axial (b) coronal image that is categorized as lung-RADS score III "benign solid nodular lesion" that is proved on follow-up for 24 months to be static small begin nodule

metastatic lesions (false negative). Five of these lesions showed significant growth in the follow-up study and 3 were accompanied by the appearance of other newly developed nodules that were not included in this study (Table 4; Figs. 1 and 2). On the other hand, 114 nodules were diagnosed as malignant lesions (score $4 \mathrm{~A}, 4 \mathrm{~B}$, and $4 \mathrm{X}$ ), with 21 of them proving to be of benign etiology (false positive; 12 inflammatory granulomas according to histopathology and 9 lesions were static in follow-up CT images up to 18 months), (Table 5, Figs. 3, 4 and 5).

Sensitivity, specificity, and accuracy of lung-RADS for final diagnosis of all studied 200 nodular lesions

Lung-RADS was found to show a high sensitivity of $92.08 \%$, specificity of $78.79 \%$, and accuracy of $85.50 \%$ with $81.58 \%$ positive predictive value and $90.70 \%$ negative predictive value in the diagnosis of pulmonary nodules in cancer patients (Table 6).

\section{Discussion}

Pulmonary metastasis is seen in $20-54 \%$ of extrathoracic malignancies [9]. Lung-RADS reporting is standardized per CT lung screening, which accounts for pulmonary nodules and other findings concerning carcinoma, as well as any significant incidental findings $[10,11]$.

The present study included 200 newly developed pulmonary nodules in 187 known cured cancer patients that were categorized by the lung-RADS calcification system into 6 sub-groups, 1-4A, 4B, and $4 \mathrm{X}$, and we considered scores of $4 \mathrm{~A}, 4 \mathrm{~B}$, and $4 \mathrm{X}$ as malignant. We found 86 lesions to be benign that were categorized as

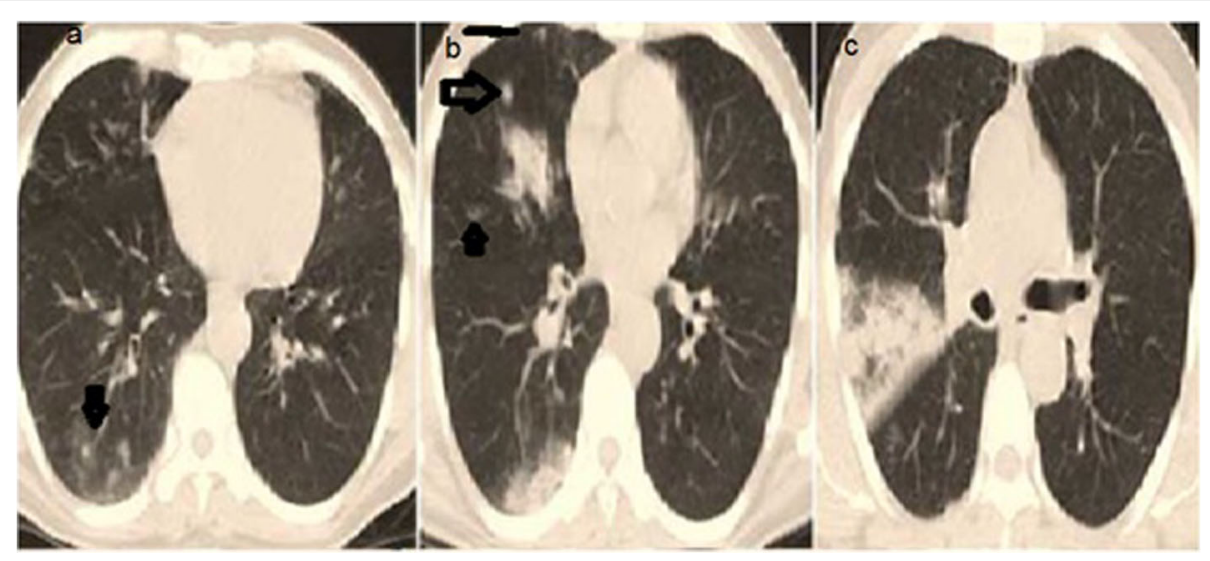

Fig. 2 "A 44-year-old female patient with a history of breast cancer."| MCCT exam axial views revealed multiple well-defined small solid nodules scattered at the periphery of the Rt. upper and lower lung lobes $(\mathbf{a}, \mathbf{b})$ that categorized as lung-RADS score 4X, "malignant metastatic solid nodular lesions" that is on follow-up after 1 month (c) proved to be benign inflammatory granuloma according to final clinical diagnosis 


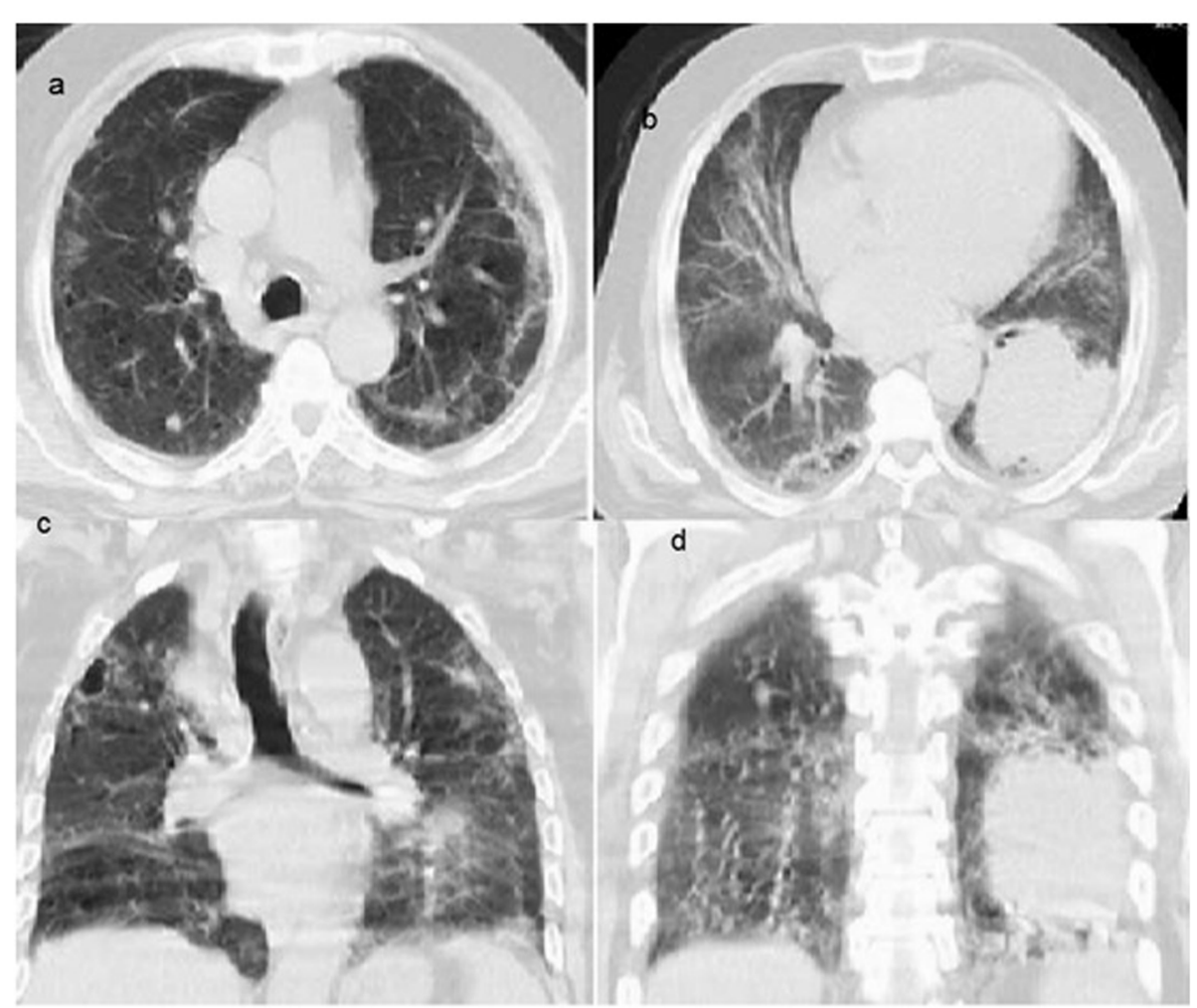

Fig. 3 "A 63-year-old male patient with a history of prostate cancer." MCCT exam revealed a well-defined small solid nodule at the Rt. lower lung lobe and a sizable soft tissue mass lesion at the Lt. lower lung lobe $(\mathbf{a}, \mathbf{b})$ axial and $(\mathbf{c}, \mathbf{d})$ coronal images that categorized as the lung-RADS score 4X, "malignant solid nodular lesions." The Lt. side lesion was pathology proved as newly developed adenocarcinoma and Rt. side considered as a metastatic lesion

lung-RADS 1-3 scores. However, 8 of them were found to be malignant metastatic lesions (false negative) in line with histopathology and follow-up results. While we diagnosed 114 nodules as malignant lesions with lungRADS 4A, 4B, and 4X scores, 21/114 proved to be of benign etiology (false positive): 6 inflammatory granulomas per histopathology results and 15 showed static appearance on LDCT chest follow-up after 18-24 months. Our findings were consistent with those of Caparica et al. [3], who conducted a similar study) on 228 patients and metastatic disease was found in 146 patients (64\%). On biopsy, 60 patients $(26.3 \%)$ were found to have a second primary lung tumor, while 22 patients $(9.6 \%)$ were found to be cancer-free. Other series have also found different rates of malignant nodules on histologic analysis. In a large study with 1104 patients undergoing PNs resection, Mery et al. [12] observed a 63\% malignancy rate in 337 patients with a previous cancer history. A lower rate by Khokhar et al. [13] discovered malignant pulmonary nodules in oncologic patients; $28 \%$ of small pulmonary

Table 5 Categorization of malignant lung nodules (lung-RADS score: 4A, B, and X). "Group II" (no. = 114 nodules)

\begin{tabular}{|c|c|c|c|c|c|}
\hline RADS score & Size & Lesion density & Lesion no. & $\%$ for all lesions & Radio-diagnosis \\
\hline \multirow[t]{3}{*}{$4 \mathrm{~A}$} & $\geq 8$ to $<15 \mathrm{~mm}$ & Solid & 9 & $4.5 \%$ & Malignant \\
\hline & $\geq 6 \mathrm{~mm}+$ solid comp. measuring $\geq 6-<8 \mathrm{~mm}$ & Part solid & 7 & $3.5 \%$ & \\
\hline & Measuring 9.3 mm & Endo-bronchial & 1 & $0.5 \%$ & \\
\hline \multirow[t]{2}{*}{$4 \mathrm{~B}$} & $\geq 15 \mathrm{~mm}$ & Solid & 32 & $16 \%$ & Malignant \\
\hline & With a solid component measuring $>8 \mathrm{~mm}$. & Part solid & 14 & $7 \%$ & \\
\hline \multirow{4}{*}{$\begin{array}{l}4 \mathrm{X} \\
4 \mathrm{~A} / 4 \mathrm{Bwith}\end{array}$} & With & Other solid lesions & 29 & $14.5 \%$ & Malignant \\
\hline & & Speculation & 11 & $5.5 \%$ & \\
\hline & & Lymphadenopathy & 4 & $2 \%$ & \\
\hline & & Growth on follow-up & 7 & $3.5 \%$ & \\
\hline
\end{tabular}




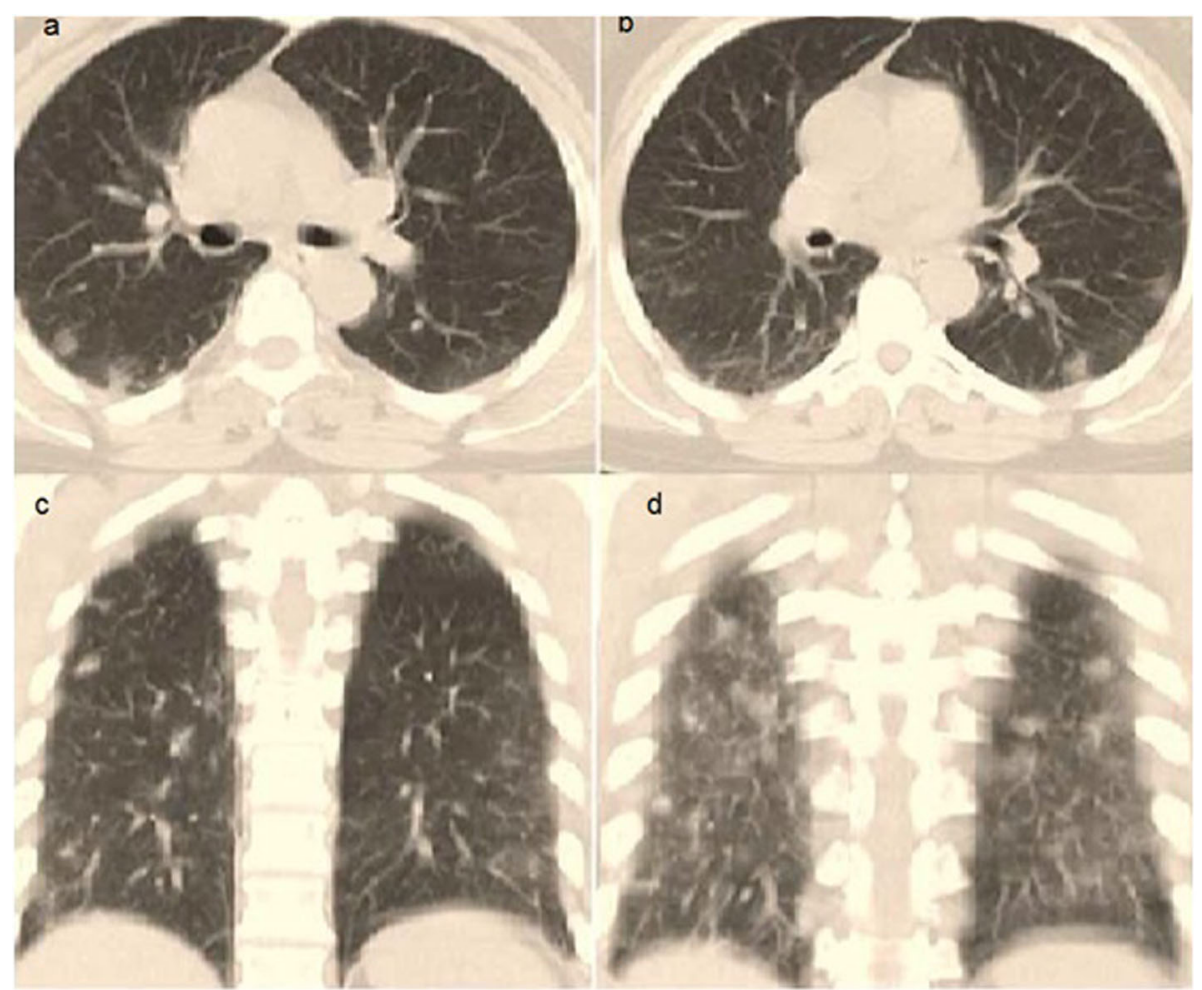

Fig. 4 "A 56-year-old female patient with a history of thyroid cancer." MCCT exam revealed multiple well-defined solid nodules scattered throughout both lung parenchyma $(\mathbf{a}, \mathbf{b})$ axial and $(\mathbf{c}, \mathbf{d})$ coronal images that are categorized as lung-RADS score $4 \mathrm{X}$, "malignant metastatic solid nodular lesions" that showed an increase in size and number in follow-up examinations which proved to be metastatic malignant nodules

nodules detected on the initial CT will increase in size, suggesting metastasis. However, Reginald et al. [14] concluded that in the majority of clinical settings, an interval increase in the size of a single pulmonary nodule is considered evidence of metastasis, this size increase tends to occur early, and follow-up CT in 3 and 6 months would be appropriate for further evaluation. On the opposite hand, Meng et al. [15] divided ground-glass nodules into three groups: benign disease (inflammations), pre-invasive group, and invasive group

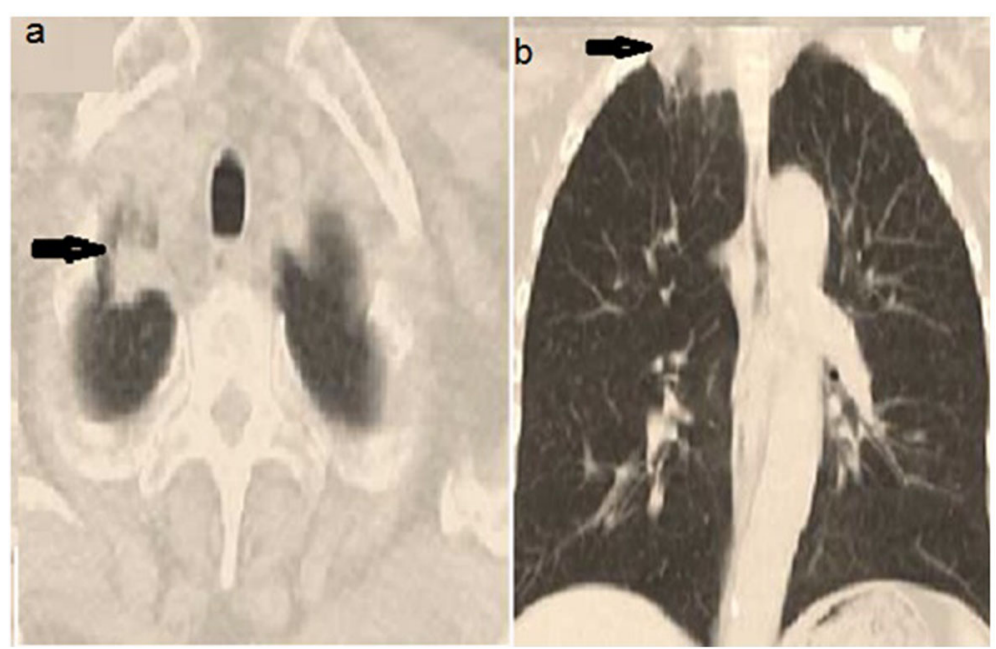

Fig. 5 "A 52-year-old female patient with a history of treated breast cancer since 1.5 years." Follow-up MCCT exam revealed an irregular welldefined semi-solid nodule at the anterior segment of the Rt. upper lung lobe (a) axial (b) coronal image that is categorized as lung-RADS score 4A "malignant nodular lesion." Pathology results newly developed small cell carcinoma 
Table 6 Sensitivity, specificity, and accuracy of lung-RADS for all studied lesions

\begin{tabular}{llllll}
\hline Modality & Sensitivity & Specificity & Accuracy & PPV & NPP \\
\hline Lung-RADS & $92.08 \%$ & $78.79 \%$ & $85.5 \%$ & $81.58 \%$ & $90.70 \%$ \\
\hline
\end{tabular}

(adenocarcinoma) in line with the pathologic findings of type IV, strongly suggesting a high likelihood of malignancy, while Kim et al. [16] stated that pulmonary subsolid nodules (SSNs) are frequently encountered within the screening CT with their main concern being lung adenocarcinoma and its precursors. Results from the National Lung Screening Trial showed that carcinoma screened by CT significantly reduced carcinoma mortality, and benign nodules were primarily confirmed by stability or shrinkage on repeat CT scans over a 2-year follow-up period [17]. Chung et al. [18] found that the typical false-positive rate was $7 \%$ for category 3 nodules, $7 \%$ for category $4 \mathrm{~A}$ nodules, and $19 \%$ for category $4 \mathrm{~B}$ nodules.

Lung-RADS may be a tool that facilitates standardized reporting and management of abnormal findings at LCS CT [8].

Our findings revealed that the lung-RADS arrangement had a high sensitivity of $92.08 \%$, specificity of $78.79 \%$, and accuracy of $85.50 \%$ in the diagnosis of pulmonary nodules in oncology patients, with $81.58 \%$ positive predictive value and $90.70 \%$ negative predictive value. These results were in agreement with McKee et al. [19], who said that ACR lungRADS reduced the general positive rate from 27.6 to $10.6 \%$. No false negatives were present within the 152 patients with > 12-month follow-up reclassified as benign. Applying ACR lung-RADS increased the positive predictive value for diagnosed malignancy in 1603 patients with follow-up from 6.9 to $17.3 \%$ and by Kessle et al. [20], who concluded that lung-RADS produced 9 false-positives and 16 false-negative findings, whereas VRC with a 5\% threshold resulted in 29 false-positives and 10 false-negative findings. Overall sensitivity and specificity for lung-RADS was $58.0 \%$ and $98.0 \%$, and for VRC with a 5\% threshold was $73.7 \%$ and $93.5 \%$, respectively $(P=.313, P=.001$, respectively).

\section{Limitations}

Due to the relatively low number of lesions and the small size of most nodules, which made biopsy and histopathology difficult, we relied on a follow-up clinical diagnosis of up to 24 months, which is acceptable in oncology patients.

\section{Conclusion}

Low-density CT with a nodule reporting system based on the lung-RADS classification system was found to be an accurate non-invasive tool to characterize and to risk stratify pulmonary nodules in oncology patients.

\section{Abbreviations}

CT: Computed tomography; Lung-RADS: Lung Reporting and Data System; LDCT: Low-dose computed tomography

\section{Authors' contributions}

The author(s) read and approved the final manuscript.

Funding

No disclosure of funding received for this work from any organization.

\section{Declarations}

Ethics approval and consent to participate

This study was done after approval from the AL-Azhar University Hospital, Faculty of Medicine Assuit, and the after patient agrees verbal consent (as the patients were not exposed to any type of surgical or intervention maneuver). This study was done from January 2019 to October 2020. The number of meeting code is 3 and the number of paper code is 7.

\section{Consent for publication}

All patients included in this research gave written informed consent to publish the data contained within this study. If the patient was less than 16 years old, deceased, or unconscious when consent for publication was requested, written informed consent for the publication of this data was given by their parent or legal guardian.

\section{Competing interests}

The authors declare that they have no competing interests.

\section{Author details}

${ }^{1}$ Radio-Diagnosis Department, Faculty of Medicine, Al-Azhar University, Assuit, Egypt. ${ }^{2}$ Radio-Diagnosis Department, Faculty of Medicine, Minia University, El-Minya, Egypt.

Received: 19 February 2021 Accepted: 2 July 2021

Published online: 16 July 2021

\section{References}

1. Loverdos K, Fotiadis A, Kontogianni C et al (2019) Lung nodules: A comprehensive review on current approach and management. Ann Thor Med 14(4)

2. Gurney JW (1993) Determining the likelihood of malignancy in solitary pulmonary nodules with Bayesian analysis. Part I. Theory Radiol 186:405-413

3. Caparica R, Perez MM, Henrique CR et al (2016) Pulmonary nodules in patients with non-pulmonary cancer: not always metastases. J Global Oncol 2(3):138-144

4. Dabrowska M, Krenke R, Korczynski P et al (2015) Diagnostic accuracy of contrast-enhanced computed tomography and positron emission tomography with 18-FDG in identifying malignant solitary pulmonary nodules. Medicine 94:e666

5. $\quad$ van SR J, Jacobs C, Th Scholten E et al (2019) Observer variability for LungRADS categorization of lung cancer screening CTs: impact on patient management. Eur Radiol 29:924-931

6. American College of Radiology website. Lung CT Screening Reporting and Data System (Lung-RADS). www.acr.org/Quality-afety/Resources /LungRADS. Accessed 21 July 2017

7. Brett WC, John PL, Carol CW et al (2018) Screening for lung cancer: lexicon for communicating with health. Care Providers AJR 210

8. Maria DM, Jeffrey PK, Lynn SB et al (2017) Lung-RADS: Pushing the Limits. RadioGraphics 37:1975-1993

9. Chung K, Jacobs C, T Scholten E et al (2017) Malignancy estimation of Lung-RADS criteria for sub-solid nodules on CT: accuracy of low and high risk spectrum when using NLST nodules. EurRadiol 27:4672-4679

10. Patel T (2018) Lung Metastases Imaging Updated: Sep 30

11. A Ferreira C, Aresta G, Pedrosa J. Classification of Lung Nodules in CT Volumes Using the Lung-RADS ${ }^{\mathrm{m}}$ Guidelines with Uncertainty Parameterization. Published in: IEEE 17th International Symposium on Biomedical Imaging (ISBI). DOI: https://doi.org/10.1109/ISBI45749.2020. 9098586 
12. Mery CM, Pappas AN, Bueno R et al (2004) Relationship between a history of antecedent cancer and the probability of malignancy for a solitary pulmonary nodule. Chest 125:2175-2181

13. Khokhar S, Vickers A, Moore MS et al (2006) Significance of non-calcified pulmonary nodules in patients with extra-pulmonary cancers. Thorax 61: 331-336

14. Reginald FM, Jeremy JE, Hisham W et al (2010) Follow-Up of Small ( $4 \mathrm{~mm}$ or Less) Incidentally Detected Nodules by Computed Tomography in Oncology Patients. A Retrospective Review. J Thorac Oncol 5:1958-1962

15. Meng Q, Ren P, Gao P et al (2020) Effectiveness and feasibility of complementary lung-RADS version 1.1 in risk stratification for pggn in ldct lung cancer screening in a chinese population. Cancer Manag Res 12:189198

16. Kim H, Min CP, Moon JK et al (2014) Pulmonary subsolid nodules: what radiologists need to know about the imaging features and management strategy. Diagn Interv Radiol 20:47-57

17. Nishio M, Sugiyama O, Yakami M et al (2018) Computer-aided diagnosis of lung nodule classification between benign nodule, primary lung cancer, and metastatic lung cancer at different image size using deep convolutional neural network with transfer learning. PLoS One. https://doi. org/10.1371/journal.pone.0200721

18. Chung K, Jacobs C, T Scholten E et al (2017) Malignancy estimation of Lung-RADS criteria for sub-solid nodules on CT: accuracy of low and high risk spectrum when using NLST nodules. Eur Radiol 27:4672-4679

19. McKee B, Regis SM, McKee A et al (2015) Performance of ACR Lung-RADS in a clinical CT lung screening program. J Am CollRadiol 12:273

20. Kessle A, Peng R, Mardakhaev E (2020) Performance of the vancouver risk calculator compared with lung-RADS in an urban, diverse clinical lung cancer screening cohort. Radiology 2:2

\section{Publisher's Note}

Springer Nature remains neutral with regard to jurisdictional claims in published maps and institutional affiliations.

\section{Submit your manuscript to a SpringerOpen ${ }^{\circ}$ journal and benefit from:}

- Convenient online submission

- Rigorous peer review

- Open access: articles freely available online

High visibility within the field

- Retaining the copyright to your article

Submit your next manuscript at $\boldsymbol{\nabla}$ springeropen.com 